\title{
From Context-Aware to Context-Based: Mobile Just-In-Time Retrieval of Cultural Heritage Objects
}

\author{
Jörg Schlötterer, Christin Seifert, Wolfgang Lutz, and Michael Granitzer \\ Media Computer Science, University of Passau, Germany \\ \{joerg.schloetterer, christin.seifert, michael.granitzer\}@uni-passau.de
}

\begin{abstract}
Cultural content providers face the challenge of disseminating their content to the general public. Meanwhile, access to Web resources shifts from desktop to mobile devices and the wide range of contextual sensors of those devices can be used to proactively retrieve and present resources in an unobtrusive manner. This proactive process, also known as just-in-time retrieval, increases the amount of information viewed and hence is a viable way to increase the visibility of cultural content. We provide a contextual model for mobile just-in-time retrieval, discuss the role of sensor information for its contextual dimensions and show the model's applicability with a prototypical implementation. Our proposed approach enriches a user's web experience with cultural content and the developed model can provide guidance for other domains.
\end{abstract}

\section{Introduction}

Recent initiatives like Europeana ${ }^{1}$ spend a huge effort on aggregating digitized museum artifacts of different institutions and providing a unified interface to access those resources. Nevertheless, users still need to be aware of those specialized portals to gain access to the tremendous collection of cultural heritage objects. Our approach is to take the content to the user, instead of taking the user to content. To this extent, we implement a just-in-time retrieval approach in a mobile setting, based on the contextual information collected by the various sensors of nowadays smartphones. These sensors capture a wide spectrum of a user's context and hence provide a great source for retrieving relevant resources and adapting to the user's needs. We align our approach along the following questions: When to retrieve and present resources to the user? What are the resources the user is interested in and can they be refined by location information of resources or users (where)?

Specifically, our contributions are the following: (i) we present a context model for just-in-time retrieval in a mobile environment, (ii) we discuss how to incorporate available sensor information into the defined context dimensions and (iii) demonstrate the applicability of the model with a prototype.

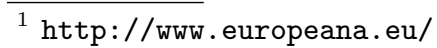




\section{Modeling Context for Mobile Just-In-Time Retrieval}

Context is usually deemed an additional dimension for personalization, either in a recommender system [2] or in information retrieval [12]. In contrast, context is the sole basis for providing recommendations in our work, resembling just-in-time retrieval [10]. Hence, we follow the rather broad definition of context as "any description of the world that can be relevant to an application" by Pete Steggles [1]. For the task of retrieving relevant resources in a mobile setting we define three abstract dimensions: (i) when, (ii) what, and (iii) where. The rational behind the three dimensions is to construct a conceptual model of the user's (potential) information need, which then can be encoded into a search query. In the following we outline how information for each dimension can be collected from either primary context, i.e. (raw/physical) sensor data (e.g. temperature), or secondary context, i.e. virtual sensors, gathering information from applications or services (e.g. message contents) or logical sensors, gathering information from physical or virtual sensors, mainly by aggregation (e.g. activities, such as walking) [3,6].

When: A user should be notified about additional resources only when it is appropriate. Interruptibility refers to a state, in which a person can be interrupted in a task without (too) negative consequences. Middleton highlights the necessity for Interface Agents to "detect when and if to interrupt the user" [8]. Noise level, observable directly by physical sensors, has been found to be a strong indicator for non-interruptibility [7]. Besides the noise level, interruptibility can be assessed according to the current situation, obtainable from logical sensors. We classify situations into trigger and blocker situations, that either initiate the recommendation process or hinder it. A combination of situations can also occur, while mostly a blocker situation will supersede one or more trigger situations.

What: One of the most valuable sources for generating search queries is textual content, which is available from the currently used application, incoming messages, notifications, etc. through virtual sensors. In order to translate the textual content into a query, keywords need to be extracted. A first step to separate stopwords and non-informative terms from those that actually convey information is named entity detection [9]. In this process, special challenges of mobile devices need to be addressed, such as short messages [11] or limited resources [4]. Given a candidate set of entities, they can be further reduced, by selecting e.g. the most salient ones [5], matching them against a user profile, etc. A very simple approach, even performable with a mobile phone's limited computing power is to choose based on frequency, i.e. how often an entity is mentioned in the text. The final set of keywords may be enriched with location information (c.f. where) and sent to the retrieval system.

Where: Location information also serves as information source to construct or refine a query. In the simplest scenario, the name of the city, the user is currently situated in, can be used as query term, in order to obtain resources about this city. Moreover, based on the current location, points of interest (POIs) nearby can be obtained, and a POI's label can be used as query term. In addition, locations identified by named entity detection (c.f. what) can also be used 
for retrieval. Cultural heritage objects can exhibit different types of locational information: the actual location of the object, i.e. the museum in whose collection it is stored, the place, from where it originated, etc. Consequently, mapping the detected locations to the appropriate query or metadata fields poses a challenge.

\section{Prototype}

To demonstrate the applicability of our proposed approach, we implemented a prototype $^{2}$ for Android mobile devices, which uses the Europeana API as search backend. Figure 1 provides a general overview of the processing chain implemented in our prototype, which is described in more detail in the following.

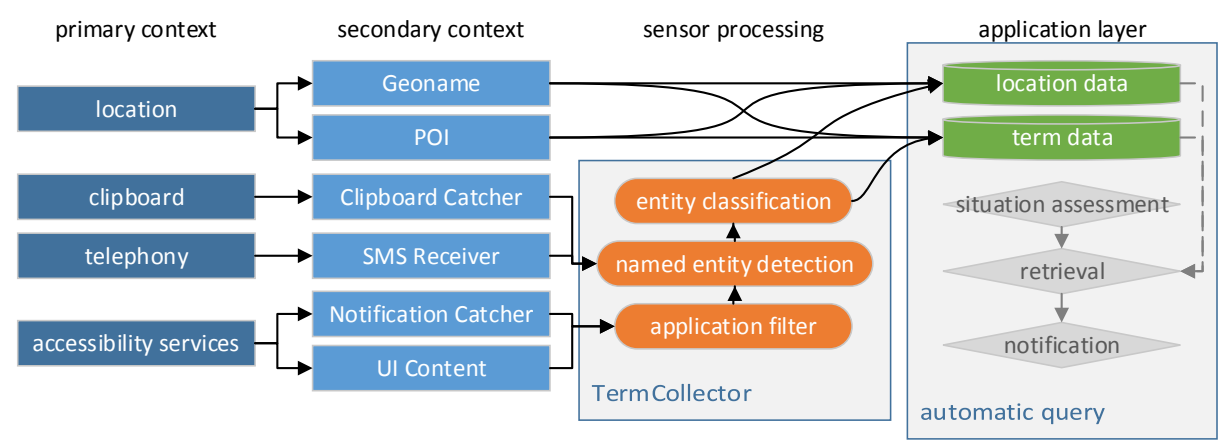

Fig. 1. Overview of the prototype's workflow.

When: We monitor incoming SMS and notifications, the content of the clipboard, content on the user interface (UI) and the user's location. Based on the latter, we obtain the geoname of the current location and nearby POIs. Activity in the just mentioned sensors is a trigger for determining when to query. After processing the context, the automatic query component evaluates the current situation(s) against a predefined set of trigger/blocker situations and, if appropriate, issues a query with the terms derived. If this query yields results, the user is notified through a ramping interface [10], featuring different stages, with each stage providing a little more information. The first stages can be ignored easily and information can be filtered early, requiring less attention from a user.

What \& Where: Context collected from notifications and the UI is filtered first by an application blacklist, in order to remove regular content such as the home screen or notifications from the Android downloader. A simple named entity detection, based on capitalization, is performed for the last four secondary context sensors in the figure and the resulting entities are classified into location or other entities. These steps are not necessary for the POI and Geoname components, as they already provide location entities. The entities obtained from all sensors are stored for further processing by the automatic query component. It

\footnotetext{
${ }^{2}$ Source and demo at http://purl.org/eexcess/components/android-app
} 
is to note, that location entities can also be used to address the what dimension as described in section 2. The Europeana API features a faceted search interface, including the facets what and where. We send the terms stored in the location data component in the where facet and those from term data in the what facet.

\section{Summary and Future Work}

We presented an approach for mobile just-in-time retrieval in the cultural heritage domain with a retrieval process purely based on contextual information and not requiring any explicit user interaction. We showed how such a process can be modeled along the contextual dimensions of when, what and where, along with a first prototype implementing this model. Even though our application focus is on cultural content, we think that the proposed model can also provide guidance for other domains. In future work, we aim to incorporate the quality of retrieved results into the decision of when to present additional resources to the user instead of relying on a binary decision based on trigger/blocker situations.

Acknowledgments The presented work was developed within the EEXCESS project funded by the European Union Seventh Framework Programme FP7/2007-2013 under grant agreement number 600601.

\section{References}

1. Abowd, G.D., Dey, A.K.: Towards a Better Understanding of Context and ContextAwareness. In: Proc. of HUC. pp. 304-307 (1999)

2. Adomavicius, G., Tuzhilin, A.: Context-aware recommender systems. In: Recommender systems handbook, pp. 217-253. Springer (2011)

3. Baldauf, M.: A survey on context-aware systems. International Journal on Ad Hoc and Ubiquitous Computing 2(4) (2007)

4. Ek, T., Kirkegaard, C., Jonsson, H., Nugues, P.: Named entity recognition for short text messages. Procedia-Social and Behavioral Sciences 27, 178-187 (2011)

5. Gamon, M., Yano, T., Song, X., Apacible, J., Pantel, P.: Identifying salient entities in web pages. In: Proc. of CIKM. pp. 2375-2380 (2013)

6. Hong, J.y., Suh, E.h., Kim, S.J.: Context-aware systems: A literature review and classification. Expert Systems with Applications 36(4), 8509-8522 (May 2009)

7. Hudson, S.E., Fogarty, J., Atkeson, C.G., Avrahami, D., Forlizzi, J., Kiesler, S., Lee, J.C., Yang, J.: Predicting Human Interruptibility with Sensors: A Wizard of Oz Feasibility Study. In: Proc. of SIGCHI (2003)

8. Middleton, S.E.: Interface agents: A review of the field. CoRR cs.MA/0203, 28 (Mar 2002)

9. Nadeau, D., Sekine, S.: A survey of named entity recognition and classification. Lingvisticae Investigationes 30(1), 3-26 (2007)

10. Rhodes, B.J.: Just-In-Time Information Retrieval. Ph.D. thesis, Massachusetts Institute of Technology (2000)

11. Ritter, A., Clark, S., Mausam, Etzioni, O.: Named entity recognition in tweets: An experimental study. In: Proc. of EMNLP. pp. 1524-1534 (2011)

12. Shen, X., Tan, B., Zhai, C.: Context-sensitive information retrieval using implicit feedback. Proc. of SIGIR p. 43 (2005) 